\title{
Chromatin remodeling regulated by steroid and nuclear receptors
}

\author{
AlAN P W OLFFE \\ Laboratory of Molecular Embroyology Nat'l Institute of \\ Child Health and Human Development NIH, Bldg, 18T, \\ Rm. 106 Bethesda, MD 20892-5431 USA. e-mail: \\ awlme@helix.nih.gov
}

\begin{abstract}
Coactivators and corepressors regulate transcription by controlling interactions between sequence-specific transcription factors, the basal transcriptional machinery and the chromatin environment. This review consider the access of nuclear and steroid receptors to chromatin, their use of corepressors and coactivators to modify chromatin structure and the implications for transcriptional control. The assembly of specific nucleoprotein architectures and targeted histone modification emerge as central controlling elements for gene expression.
\end{abstract}

Key words: Steroid receptor, nuclear receptor, coactivator, corepressor, transcriptional activation, nucleosome positioning, histone modification, transcriptional repression.

\section{INTRODUCTION}

Chromatin structural transitions directed by nuclear and steroid hormone receptors are closely associated with gene regulation at the transcriptional level. Recent advances have defined how receptors gain access to nucleosomal DNA and then target the remodeling of chromatin structure. Remodeling of chromatin involves the receptor-mediated recruitment of molecular machines and enzymatic activities known as corepressors or coactivators that alternately stabilize or destabilize histoneDNA interactions. These complex multicomponent proteins also interact directly with the basal transcriptional machinery. The combination of their direct effects on the assembly and stability of the pre-initiation complex and their manipulation of the chromatin environment within which it functions to control transcription. The 
Chromatin and transcriptional control

purpose of this review is to summarize these observations, and describe model systems that illustrate how hormone receptors might make use of chromatin remodeling to regulate transcription.

\section{DNase I sensitivity of chromatin is dependent on hormone receptors: the examples of the ovalbumin and vitellogenin genes}

The enzyme deoxyribonuclease I (DNase I) is a useful probe of chromatin structure. Early studies found that the ovalbumin gene was 2 to 3 fold more readily digested by DNase I than bulk chromatin in nuclei isolated from chicken oviduct[14]. DNase I sensitivity was extended over a $100 \mathrm{~Kb}$ domain of DNA even in the absence of transcription[5,6]. The increased access of DNase I to this chromatin domain might be explained by the presence of clusters of regulatory proteins bound to DNA which are detected as DNase I hypersensitive sites (DHSs), other reasons might include the targeted post-translational modification of histones (see later). DHSs are sites where DNase I introduces a double-stranded break in DNA, they are a universal feature of transcriptionally competent genes in chromatin[7, 8]. Burch and Weintraub demonstrated that a series of DHSs were present in the promoter of the VTGII gene in liver[9], one site was induced by the estrogen receptor[9, 10], the other DHSs are caused by binding of liver specific transcription factors and the basal transcriptional machinery [11]. High resolution genomic footprinting results suggest that specific nucleosomal structures exist between sites of transcription factor association.

The Xenopus vitellogenin B1 gene promoter has been used to explore the functional roles of specific chromatin structures. A liver specific, estrogen responsive in vitro transcription system $[12,13]$ was used to establish that nucleosome assembly and the transcription factor NF1 acted synergistically to facilitate transcription from the vitellogenin B1 promoter in vitro[14]. A positioned nucleosome is assembled on this promoter between the estrogen response element at -300 and proximal promoter elements at -120 [15]. Both intrinsic DNA curvature and interactions of NF1 with histone H3 helps to mediate the exact positioning of the histone octamer with respect to regulatory DNA[16]. This specific chromatin structure potentiates transcription over 100 fold relative to the assembly of non-specific structures (Fig 1).

The vitellogenin genes provide a useful system for examining the relationship between transcription and chromatin structure, this is because they can be repeatedly inducted by multiple exposures to estrogen[17]. Estrogen is rapidly metabolized in the male leading to only the transient activation of vitellogenin gene transcription following a single exposure to hormone[18, 19]. When the vitellogenin genes are first activated in male liver following addition of estrogen there is an increase in general DNase I sensitivity, as well as the appearance of DHSs[18]. Subsequent additions of 

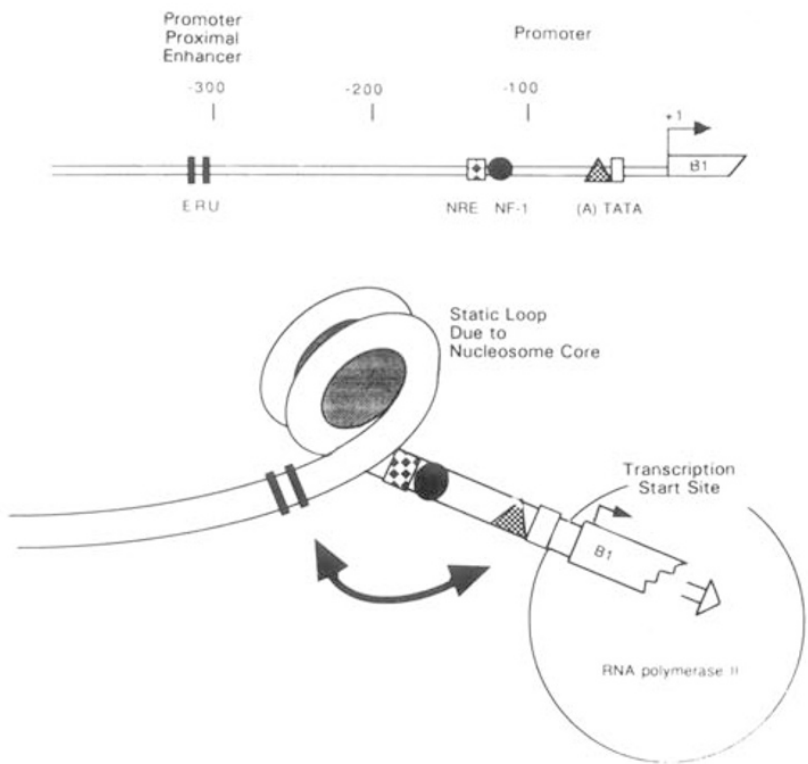

activation

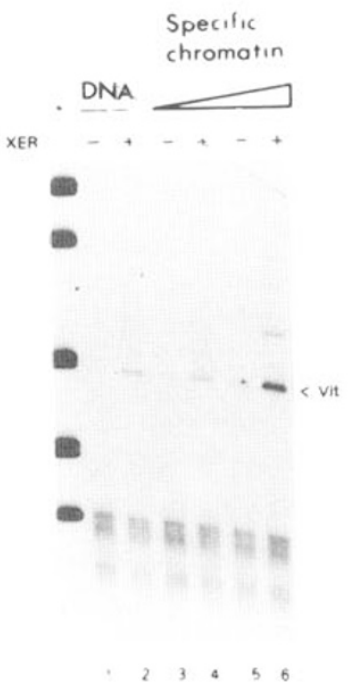

\section{repression}

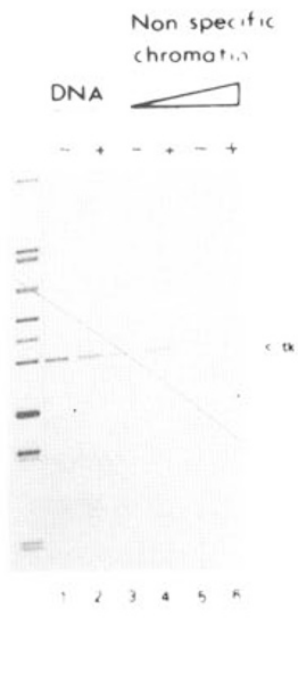

Fig 1. Role of a static loop created by a nucleosome including part of the Xenopus vitellogenin B1 promoter in potentiating transcription. The positioning of a nucleosome in the region-300 to -140 relative to the site of initiation of transcription $(+1)$ is shown by the two coils of DNA around the shaded ellipsoid. The folding of the DNA around the core histones creates a static loop which is proposed to facilitate the interaction of the estrogen receptor complex with the transcription factors present at the proximal promoter elements (black arrow). Transcription from a specific chromatin template that positions nucleosomes increases with increasing number of nucleosomes. Transcription from a non- specific chromatin template in which nucleosomes are not positioned decreases with increasing numbers of nucleosomes. (Reproduced, with permission, from Schild, C. et al., EMBO J. 1993, 12, 423. Copyright 1993 by IRL Press Ltd.). 
estrogen lead to a more vigorous and sustained transcription of the vitellogenin genes[19]. Chromatin structural transitions are not essential for this 'memory' of prior hormonal stimulation, instead autoregulatory activation of estrogen receptor gene expression appears to have the major role[20]. Cell division events correlate with the progressive loss of estrogen induced chromatin structural transitions suggesting that DNA replication and/or mitosis return chromatin structure to the ground state[21]. The estrogen receptor requires only the DNA binding domain to gain access to chromatin in Saccharomyces cerevisiae[22, 23]. This property of the receptor is similar to the capabilities of other sequence-specific transcription factors that find their binding sites in yeast chromatin without difficulty[24]. This may reflect unique properties of yeast chromatin, such as constitutive hyperacetylation of the core histones and the presence of a linker histone of unusual structure[25, 26]. Addition of an activation domain to the estrogen receptor leads to more dramatic rearrangements of chromatin structure[27, 28]. This suggests that the receptor recruits chromatin remodeling machines such as the yeast SWI/SNF complex[29] and/or the histone acetyltransferases[30]. Exactly how these molecular machines function to regulate transcription is a topic of an intense current research effort (see later).

The experimental examples that we have presented using the ovalbumin and vitellogenin genes have led to several important discoveries including: 1) that specific chromatin structures in responsive genes exist prior to the addition of hormone, 2) that specific structural transitions occur following hormone addition both in the promoter and over the chromatin domain containing responsive genes; and 3) that these transitions are reversible. Recent progress has substantiated and further defined the functional role of these chromatin remodeling events.

\section{Chromatin remodeling on the mouse mammary tumor virus long terminal repeat (MMTV LTR) induced by the glucocorticoid recep- tor}

The promoters of acutely inducible genes such as those under hormonal control are organized into specific regulatory nucleoprotein architectures[31, 32, 33]. These regulatory structures are not static, but are dynamic with respect to both proteinprotein and protein- DNA interactions. The MMTV LTR is incorporated into six positioned nucleosomes in both episomes and within a mouse chromosome. These nucleosomes serve to prevent the basal transcriptional machinery associating with the promoter in the absence of glucocorticoids[34]. Induction of transcription by glucocorticoids requires binding of the glucocorticoid receptor (GR) to the LTR, disruption of the local chromatin structure and the assembly of a transcription complex over the TATA box[34, 35].

GR binding to the MMTV LTR occurs at DNA sequences known as glucocorticoid response elements (GREs) contained within a key regulatory nucleosome. This nucleosome has been determined to have distinct translational positions with respect 
to DNA sequence, histones contacting DNA either between -188 to $-45[36]$ or at -219 to $-76[37]$ or at -221 to -78 [38] relative to the start site of transcription $(+1)$. These in vitro nucleosome positions compare favourably with those determeined in vivo[39, 40].

In all of these instances the organization of the individual GREs with respect to the surface of the histone octamer will be similar. The heterogeneity in translational position appears to reflect nucleosome mobility[41, 42] leading the histone octamer to sample multiple translational positions along the double-helix. Nucleosome mobility can be an important contributory factor in facilitating transcription[43]. The DNA sequence containing the GREs has regions of intrinsic flexibility and curvature that direct the histones to bind it in a particular way[41, 44]. Two of the GREs are separated by $92 \mathrm{bp}$ which places them together on one side of the nucleosome. This proximity might facilitate both the binding and subsequent activity of GR[45]. The display of DNA binding sites on the surface of the nucleosome can actually promote the formation of a functional transcription complex[46, 47].

Experiments that compare the affinity of the GR for a recognition element as free DNA compared to one facing towards solution in the nucleosome at different translational positions, show that binding affinity is reduced 3 to ll-fold in the nucleosome[48]. This is a remarkably small reduction compared to the complete absence of binding when the recognition element is facing towards the histones[49]. This suggests that the key variable determining the accessibility of the GR to its recognition elements within the nucleosome is not the translational position of the recognition element, but the rotational position of the DNA sequence with respect to the surface of the histones (see also refs. 8 and 50).

The GR is well suited to interact specifically with nucleosomal DNA. GR binds to DNA using a domain containing two zinc fingers: an an $\alpha$-helix in one of the two fingers interacts with a short 6 bp region in the major groove of the double helix, while the other finger is involved in proteinprotein interaction[51]. Surprisingly, association of the GR with the nucleosome containing its binding site appears to have no effect on the integrity of the structure in vitro, unlike the apparent consequence in vivo. Binding of the promoter- specific transcription factor NF1 is facilitated by the GR in vivo, however this does not occur in vitro on nucleosomal templates[52, 53]. Certain nuclear components that presumably facilitate chromatin structural changes in vivo have so far been lacking in the in vitro system.

DNA replication is one event that is certain to disrupt chromatin and provide access of transcription factors to their cognate sequences. However, DNA replication is not required for chromatin disruption and transcriptional activation of the MMTV LTR $[3,5]$. Schutz, Richard- Foy and colleagues have extended these studies by examining not only the initial disruption of chromatin structure over the enhancer of the rat tyrosine amino transferase gene (TAT) following induction of GR binding, but also the reformation of normal chromatin structure on hormone withdrawal. Both of these changes occur within a few hours, implying that neither disruption 
nor reassembly of nucleosomes is dependent on DNA replication[54, 55, see also ref. 56]. If replication is not involved in chromatin rearrangement, nucleosomes must be disrupted in some alternative way. Trans-acting factors might displace histones from nucleosomes directly. Alternatively they might wait either for histones to exchange passively out of chromatin or for DNA to spontaneously unravel from association with the histones before binding to their recognition sequences[57, 58]. The characterization of chromatin remodeling enzymes and machines at the genetic and biochemical level would make these passive mechanisms appear unlikely, even though they clearly explain some in vitro results[59]. In most assays, nucleases are used to examine the incorporation of specific promoter elements into nucleosomes. It is possible that nucleosomes that have altered their position or composition would lose sharp boundaries to nuclease protection even though DNA could remain associated with histones[60]. ProteinDNA cross- linking reagents and antibodies against histones are being used to explore this possibility.

Histone $\mathrm{H} 1$ and histone $\mathrm{H} 2 \mathrm{~A} / \mathrm{H} 2 \mathrm{~B}$ are known to exchange readily in and out of chromatin under physiological conditions[61, 62]. A partial disruption of the nucleosome in addition to histone acetylation (see later) seems by far the most likely mechanism by which trans- acting factors might gain access to DNA in chromatin. Removal of histone $\mathrm{H} 1$ from the nucleosome might facilitate some mobility of the contacts made by the histone octamer with DNA[63]. This local nucleosome sliding could facilitate transcription factor access to recognition sites otherwise constrained within the nucleosome[43, 64-66]. A certain amount of DNA folding would occur even in the absence of these proteins. The capacity to reassemble a complete nucleosome would also remain in the residual interaction of histones H3/H4 with DNA[67].

The proteins that GR recruits to the MMTV chromatin include the mammalian homologues of the SWI (Switch) 1, 2, 3 proteins of S. cerevisiae [68, 69]. The exact mechanism whereby they disrupt chromatin is unknown, but they initiate a chain of events that cause the removal of histone H1 from the linker DNA in MMTV chromatin[70] and a substantial increase in the accessibility of the DNA that is within the positioned nucleosomes to nucleases[31]. The positioning of the nucleosomes is likely to have an important role in the displacement of histone $\mathrm{H} 1$, since the linker region between the nucleosomes contains the binding sites for histone H1, NF1 and the octamer factor. It is possible that histone $\mathrm{H} 1$ and the transcription factors might compete for binding to this linker region. In any event the transcription factors NF1, the octamer factor and TFIID that lie in the linker DNA and at the periphery of the positioned nucleosomes are recruited to their binding sites in this disrupted chromatin [34] and assemble an active transcription complex. Transcriptional activation by GR is only transient and after a few hours the basal transcription complex, NF1, octamer factor and GR are displaced from the MMTV LTR and their binding sites are reincorporated into the positioned nucleosomes and the promoter repressed[71]. The molecular mechanisms responsible for displacement of the transcription factors have not been determined; however, these results indicate that both transcription 
factor and histone complexes with promoters are likely to be dynamic.

Although targeted histone acetylation is yet to be documented for MMTV chromatin in response to the presence of hormone- bound GR, it provides an attractive mechanism to locally disrupt chromatin and facilitate the function of the basal transcriptional machinery. Consistent with such a mechanism, Beato and colleagues have found that moderate increases in histone acetylation activate the mouse mammary tumor virus promoter and remodel its chromatin structure[72].

The MMTV LTR provides a powerful example of the remarkable hierarchy of nucleoprotein complexes and their restructuring during transcriptional activation. The rotational positioning of regulatory DNA on the histone octamer surface, the translational positioning of the histone octamer with respect to DNA sequence, and the histones present in MMTV LTR chromatin all contribute to the regulation of glucocorticoid receptor mediated gene activation. However it is not only the activation of transcription that illustrates the highly structured and dynamic nature of the MMTV LTR nucleoprotein complex (Fig 2), but also the subsequent inactivation of the transcription process in the presence of ligand-bound receptor (71). Inactivating genes is equally important to the regulation of transcription as activation itself. The dual contribution of gene activation and repression to regulatory phenomena is best illustrated by the thyroid hormone receptor.
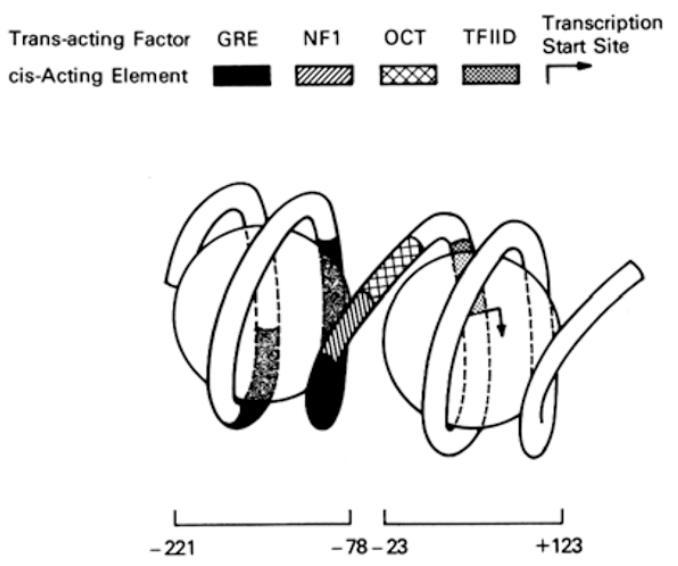

Fig 2. The organization of the MMTV LTR into a specific chromatin structure. Nucleosome positions and key recognition elements for transacting factors are indicated. The hooked arrow indicates the start site of transcription. Numbers indicate base pair positions of DNA that associated with histones relative to this site.

\section{Nucleosome positioning, reversible histone modification, transcrip- tional repression and activation: the example of the thyroid hormone receptor}

The thyroid hormone receptor was established as an integral component of chromatin from pioneering studies in the lab of Herb Samuels[73, 74]. Recent evidence suggests that the thyroid hormone receptor regulates transcription through interaction with a variety of macromolecular complexes including those that re- 
model chromatin. Thyroid hormone receptor binding proteins include heterodimeric partners[75-77], the basal transcriptional machinery[78, 79], transcriptional intermediary factors (TIFs)/coactivators[80-83] and corepressors[84-86]. Transcriptional coactivators include p300 and associated proteins such as PCAF which possess histone acetyltransferase activity[82, 87-90]. Transcriptional corepressors include NCoR [84] and SMRT[85] and associated proteins such as SIN3 and histone deacetylase[91-93]. The observations that transcriptional activation is associated with recruitment of histone acetyltransferase, and repression with recruitment of histone deacetylase leads to the hypothesis that the modification of chromatin structure targeted by the thyroid hormone receptor might contribute to transcriptional control.

Acetylation of the core histones correlates with gene activity[94 -97]. Nucleosomes containing acetylated core histones are accessible to the transcriptional machinery and much less repressive both to transcription initiation and elongation $[98,99]$. The discovery that the S. cerevisiae coactivator GCN5p has histone acetyltransferase activity led to the recognition of that targeted acetylation of histones might occur in metazoans[100]. A human homolog of GCN5p known as p300/CBP associated factor (PCAF) acetylates histones[88], as does p300/CBP itsel[89]. p300/CBP serves as an integrator to mediate regulation by a wide variety of sequence-specific transcription factors[S2] including steroid and nuclear hormone receptors, c- Jun/vJun, cMyb/vMyb, c-Fos and MyoD[101]. The capacity of TR/RXR to bind to p300/CBP suggests that targeted acetylation of the core histones and potentially of TFIIE and F might contribute to gene activation. This hypothesis is made even more attractive by the definition of histone deacetylase as a targeted transcriptional repressor.

The purification of the mammalian histone deacetylase and the recognition of the similarities to $S$. cerevisiae RPD3p[102] has provided considerable insight into transcriptional repression in metazoans. The first direct evidence for mammalian homologs of RPD3p being involved in transcriptional repression came from two hybrid screens indicating that the transcriptional regulatory factor YY1 interacted with mouse and human RPD3p[103]. The fusion of mammalian RPD3p to a targeted DNA binding domain directed transcriptional repression by more than 10 fold. Mutations in a glycine rich domain of YY1 that directs binding to RPD3p could abolish transcriptional repression by YY1 suggesting that YY1 negatively regulates transcription by tethering RPD3p. YY1 is a mammalian zinc- finger transcription factor[104] that is proposed to regulate cell growth and differentiation[105].

Eisenman and colleagues identified two mammalian proteins mSin3A and mSin3B that have striking homology to $S$. cerevisiae Sin3p including the four paired amphipathic helix (PAH) domains (106). These investigators established that the mSIN3 proteins interact with the mammalian histone deacetylases, mSIN3 and the mammalian histone deacetylases coimmunoprecipitate[107]. The third PAH domain of mSIN3 interacts with the mammalian RPD3p homologs and can confer transcriptional repression when attached to a DNA binding domain. More subtle mutational analysis suggests that the cell transformation and transcriptional repression depend 
on distinct domains of the mSIN3 proteins[91]. However an active role for histone deacetylation in transcriptional control is demonstrated by the use of deacetylase inhibitors such as Trichostatin A that abolish the capacity of mSIN3- deacetylase complexes to repress transcription[108]. The existence of a conserved transcriptional repression mechanism that utilizes SIN3p and histone deacetylase emphasizes the significance of the chromatin environment for transcriptional control. Histone deacetylation directs the assembly of a stable repressive chromatin structure.

A role for chromatin had already been established in the control of transcription by the thyroid hormone receptor[8, 109, 110]. These studies provide a useful example of how the histones can contribute to gene regulation. The assembly of mini-chromosomes within the Xenopus oocyte nucleus has been used to examine the role of chromatin in both transcriptional silencing and activation of the Xenopus TR $\beta$ A promoter. Transcription from this promoter is under the control of thyroid hormone and the thyroid hormone receptor [111], which exists as a heterodimer of TR and RXR. Microinjection of either single-stranded or double-stranded DNA templates into the Xenopus oocyte nucleus offers the opportunity for examination of the influence on gene regulation of chromatin assembly pathways that are either coupled or uncoupled to DNA synthesis[112]. The staged injection of mRNA encoding transcriptional regulatory proteins and of template DNA offers the potential for examining the mechanisms of transcription factor-mediated transcriptional activation of promoters within a chromatin environment. In particular, it is possible to discriminate between pre- emptive mechanisms in which transcription factors bind during chromatin assembly to activate transcription, and post-replicative mechanisms in which transcription factors gain access to their recognition elements after they have been assembled into mature chromatin structures. TR/RXR heterodimers bind constitutively within the minichromosome, independently of whether the receptor is synthesized before or after chromatin assembly. Rotational positioning of the TRE on the surface of the histone octamer allows the specific association of the TR/RXR heterodimer in vitro[8]. The coupling of chromatin assembly to the replication process augments transcriptional repression by unliganded TR/RXR without influencing the final level of transcriptional activity in the presence of thyroid hormone.

The molecular mechanisms by which the unliganded thyroid hormone receptor makes use of chromatin in order to augment transcriptional repression also involve mSin3 and histone deacetylase[91, 92]. The unliganded thyroid hormone receptor and retinoic acid receptor bind a corepressor NCoR[84]. NCoR interacts with Sin3 and recruits the histone deacetylase[91, 92]. All of the transcriptional repression conferred by the unliganded thyroid hormone receptor in Xenopus oocytes[109, 110] can be alleviated by the inhibition of histone deacetylase using Trichostatin A. This is indicative of an essential role for deacetylation in establishing transcriptional repression in a chromatin environment.

The addition of thyroid hormone to the chromatin bound receptor leads to the 
disruption of chromatin structure[109, 110]. Chromatin disruption is not restricted to the receptor binding site and involves the reorganization of chromatin structure in which targeted histone acetylation by the PCAF and p300/CBP activators may have a contributory role[88, 89]. It is possible to separate chromatin disruption from productive recruitment of the basal transcription machinery in vivo by the deletion of regulatory elements essential for transcription initiation at the start site and by the use of transcriptional inhibitors[109, 110]. Therefore chromatin disruption is an independent hormone-regulated function targeted by DNA- bound thyroid hormone receptor. It is remarkable just how effectively the various functions of the thyroid hormone receptor are mediated through the recruitment of enzyme complexes that modify chromatin. These results provide compelling evidence for the productive utilization of structural transitions in chromatin as a regulatory principle in gene control.

The genetic, biochemical and cell biological evidence that we have outlined provides a substantial rationale for considering chromatin structural proteins as integral components of the transcriptional machinery. It is important to recognize that chromatin structure is not necessarily static and obstructive to transcription but provides a means of display for the DNA template that determines function. Variation in the quality of histone-DNA interactions and in the three- dimensional path of the double helix can directly influence transcription[15, 99]. Conformation is a well known determinant of enzymatic activity, alterations in chromatin conformation may well determine transcriptional activity.

The facts that: 1) core histone acetylation greatly facilitates the access of transcription factors to DNA in a nucleosome, 2) that transcriptional repressors recruit histone deacetylases, and 3) that transcriptional coactivators are histone acetyltransferases leads to a model for transcriptional regulation in which the recruitment of repressors could direct the local stabilization of repressive histone- DNA interactions and where the recruitment of activators could destabilize these interactions. Repressive nucleosomes might prevent either the association or function of the basal transcriptional machinery on a particular promoter. However it is important to note that certain transcriptional regulators such as the thyroid hormone receptor can bind to their recognition elements in a nucleosome even when the histones are deacetylated[109, 110], and that nucleosome assembly is not always repressive[15].

The recruitment of histone deacetylase by chromatin bound repressors will potentially eliminate basal levels of histone acetylation and impede the recruitment or function of the basal transcriptional machinery. Targeted acetylation provides a means of allowing the basal machinery to displace nucleosomes, assemble a functional transcription complex and never have to deal with chromatin again. For example we can propose three steps in the regulation of transcription by thyroid hormone receptor: 1) thyroid hormone receptor binds to chromatin on the surface of a positioned nucleosome and facilitates the assembly of a repressive chromatin structure, 2) in response to hormone, the receptor recruits molecular machines or enzymes that 
disrupt local chromatin structure, 3) the hormone- bound receptor and associated activators facilitate the recruitment and activity of the basal transcriptional machinery to further activate transcription. Additional interesting possibilities include the regulated association and activity of histone acetyltransferases and deacetylases within a common complex. In this way transcriptional activity could be continually modulated through variation in chromatin conformation.

\section{CONCLUSIONS}

Nuclear and steroid hormone receptors function effectively within a chromatin environment. In many ways the function of these receptors depends upon their ability to manipulate chromatin structure towards instability or stability. These events occur dynamically within a structure that allows receptor association and that can reversibly accomodate the conformational and compositional transitions induced by the receptor. Most attention in this research field is currently focussed on the chromatin remodeling machines that activate or repress transcription. It is nevertheless essential to understand the structures that exist before remodeling, the remodeled state, and those that are present when the gene is inactivated. Moreover attention should be focussed not only on histone-DNA interactions but also on the activities of the basal transcriptional machinery in the context of chromatin. The principal function of receptors may well be to facilitate the conversation between the basal transcriptional machinery and the chromatin environment in which it functions.

\section{REFERENCES}

[1] O'Malley BW, Towle HC, Schwartz RJ. Regulation of gene expression in eukaryotes. Ann Rev Gent 1977; 11:239-75.

[2] Lawson GM, Tsai M J, O'Malley BW. Deoxyribonuclease I sensitivity of the non-transcribed sequences flanking the $5^{\prime}$ and 3 ' ends of the oromucoid gene and the ovalbumin and its related $\mathrm{X}$ and $\mathrm{Y}$ genes in hen oviduct nuclei. Biochemistry 1980; 19:4403-41.

[3] Lawson GM, Knoll B J, March C J, Woo SL, Tsai M J, O'Malley BW. Definition of 5' and 3' structural boundaries of the chromatin domain containing the ovalbumin multigene family. $J$ Biol Chem 1982; 257:1501-7.

[4] Anderson JN, Vanderbilt JN, Lawson GM, Tsai M J, O'Malley BW. Chromatin structure of the ovalbumin gene family in the chicken oviduct. Biochemistry 1983; 22:21-30.

[5] Stumph WE, Baez M, Beattie WG, Tsai M J, O'Malley BW. Characterization of deoxyribonucleic acid sequences at the 5 ' and 3 ' borders of the 100 kilobase pair ovalbumin gene domain. Biochemistry 1983; 22:306-15.

[6] Stumph WE, Baez M, Lawson GM, Tsai M J, O'Malley BW. Higher-order structural determinants for expression of the ovalbumin gene family. Ciba Found Symp 1983; 98:80-95.

[7] Elgin SCR. The formation and function of DNaseI hypersensitive sites in the process of gene activation. J Biol Chem 1988; 263:19259-62.

[8] Wong J, Li Q, Levi B-Z, Shi Y-B, Wolffe AP. Structural and functional features of a specific nucleosome containing a recognition element for the thyroid hormone receptor. EMBO J 1997; (in press).

[9] Burch JB, Weintraub H. Temporal order of chromatin structural changes associated with acti- 


\section{Chromatin and transcriptional control}

vation of the major chicken vitellogenin gene. Cell 1983; 33:65-76.

[10] Burch JB, Evans MI, Friedman TM, O'Malley PJ. Two functional estrogen response elements are located upstream of the major chicken vitellogenin gene. Mol Cell Biol 1988; 8:1123-31.

[11] Philipsen JN, Hennis BC, Ab G. In vivo footprinting of the estrogen-inducible vitellogenin II gene from chicken. Nucleic Acids Res 1988; 16:9663-76.

[12] Corthesy B, Cardinaux J-R, Claret F-X, Wahli W. An NF1- like activity and a liver-specific repressor govern estrogen-regulated in vitro transcription from the Xenopus vitellogenin B1 promoter. Mol Cell Biol 1989; 9:5548-62.

[13] Corthesy B, Hipskind R, Theulaz I, Wahli W. Estrogen-dependent in vitro transcription from the vitellogenin promoter in liver nuclear extracts. Science 1988; 239:1137-9.

[14] Corthesy B, Leonnard P, Wahli W. Transcriptional potentiation of the vitellogenin B1 promoter by a combination of both nucleosome assembly and transcription factors: an in vitro dissection. Mol Cell Biol 1990; 10:3926-33.

[15] Schild C, Claret F-X, Wahli W, Wolffe AP. A nucleosome- dependent static loop potentiates estrogen-regulated transcription from the Xenopus vitellogenin B1 promoter in vitro. EMBO J 1993; 12:423-33.

[16] Alevizopoulos A, Dusserre Y, Tsai-Pflulgfelder M, Von Der Weid T, Wahli W, Mermod N. A proline-rich TGF- $\beta$-responsive transcriptional activator interacts with histone H3. Genes Dev 1995: 9:3051-66.

[17] Tata JR, Smith DF. Vitellogenesis: a versatile model for hormonal regulation of gene expression. Recent Prog Hormone Res 1979; 35:47-90.

[18] Williams JL, Tata JR. Simultaneous analysis of conformation and transcription of A and B roups of vitellogenin genes in male and female Xenopus during primary and secondary activation by estrogen. Nucl Acids Res 1983; 11:1151-66.

[19] Wolffe AP, Tata JR. Coordinate and non-coordinate estrogen-induced expression of A and B groups of vitellogenin genes in male and female Xenopus hepatocytes in culture. Eur J Biochem 1983; 130:365-72.

[20] Barton MC, Shapiro DJ. Transient administration of estradiol- 17 beta establishes an autoregulatory loop permanently inducing estrogen receptor mRNA. Proc Natl Acad Sci USA 1988; 85:7119-23.

[21] Burch JB, Evans, MI. Chromatin structural transitions and the phenomenon of vitellogenin gene memory in chickens. Mol Cell Biol 1986; 6:1886-93.

[22] Gilbert DM, Losson R, Chambon P. Ligand dependence of estrogen receptor induced changes in chromatin structure. Nucl Acids Res 1992; 20:4525-31.

[23] Pham TA, Ellist JF, Nawaz Z, McDonnell DP, Tsai M J, O'Malley BW. Antiestrogen can establish non productive receptor complexes and alter chromatin structure at target enhancers. Proc Natl Acad Sci USA 1991; 88:3125-9.

[24] Morse RH. Nucleosome disruption by transcription factor binding in yeast. Science 1993; 262:1563-6.

[25] Clark L J, O'Neill LP, Turner BM. Selective use of H4 acetylation sites in the yeast Saccharomyces cerevisiae. Biochem J 1993; 294:557-61.

[26] Landsman D. Histone H1 in Saccharomyces cerevisiae: a double mystery solved. Trends Biochem Sci 1996; 21:287-8.

[27] Pham TA, Hwung Y-P, McDonnell DP, O'Malley BW. Transactivation functions facilitate the disruption of chromatin structure by estrogen receptor derivaties in vivo. J Biol Chem 1991; 266:18179-87.

[28] Stafford GA, Morse RH. Chromatin remodeling by transcriptional activation domains in a yeast episome. J Biol Chem 1997; 272:11526-34.

[29] Chiba H, Muramatsu M, Nomoto A, Kato H. Two human homologs of Saccharomyces cerevisiae SWI2/SNF2 and Drosophila brahma are transcriptional coactivators cooperating with the estrogen receptor and the retinoic acid receptor. Nucl Acids Res 1994; 22:1815-20. 
[30] Hanstein B, Eckner R, DiRenzo J, Halachmi S, Liu H, Searoy B, Kurokawa R, Brown M. p300 is a component of an estrogen receptor coactivator complex. Proc Natl Acad Sci USA 1996; 93:11540-5.

[31] Zaret KS, Yamamoto KR. Reversible and persistent changes in chromatin structure accompany activation of a glucocorticoid dependent enhancer element. Cell 1984; 38:29-38.

[32] Wolffe AP. Architectural transcription factors. Science 1994; 264:1100-1.

[33] Wolffe AP. Nucleosome positioning and modification: chromatin structures that potentiate transcription. Trends Biochem Sci 1994; 19:240-4.

[34] Archer TK, Lefebvre P, Wolford RG, Hager GL. Transcription factor loading on the MMTV promoter: a bimodal mechanism for promoter activation. Science 1992; 255:1573-6.

[35] Archer TK, Cordingley MG, Marsaud V, Richard-Foy H, Hager GL. Steroid transactivation at a promoter organized in a specifically positioned array of nucleosomes. In Proceedings: Second International CBT Symposium on the Steroid/Thyroid Receptor Family and Gene Regulation. Springer Verlage, Berlin, 1989: pp 221-38.

[36] Pina B, Barettino D, Truss M, Beato M. Structural features of a regulatory nucleosome. J Mol Biol 1990; 216:975-90.

[37] Perlmann T, Wrange O. Specific glucocorticoid receptor binding to DNA reconstituted in a nucleosome. ЕMBO J 1988; 7:3073-83.

[38] Archer TK, Cordingley MG, Wolford RG, Hager GL. Transcription factor access is mediated by accurately positioned nucleosomes on the mouse mammary tumor virus promoter. Mol Cell Biol 1991; 11:688-98.

[39] Richard-Foy H, Hager GL. Sequence- specific positioning of nucleosomes over the steroid- inducible MMTV promoter. EMBO J 1987; 6:2321-8.

[40] Fragoso G, John S, Robertis S, Hager GL. Nucleosome positioning on the MMTV LTR from the frequently-biased occupancy of multiple frames. Genes Dev 1995; 9:1933-47.

[41] Roberts MS, Fragoso G, Hager GL. The MMTV LTR B nucleosome adopts multiple translational and rotational positions during in vitro reconstitution. Biochemistry 1995; 34:12470-80.

[42] Meersseman G, Pennings S, Bradbury EM. Mobile nucleosomes- a general behavior. EMBO J 1992; 11:2951-9.

[43] Ura K, Hayes J J, Wolffe AP. A positive role for nucleosome mobility in the transcriptional activity of chromatin templates: resitriction by linker histones. EMBO J 1995; 14:3752-65.

[44] Pina B, Bruggemeier U, Beato M. Nucleosome positioning modulates accessibility of regulatory proteins to the mouse mammary tumor virus promoter. Cell 1990; 60:719-31.

[45] Perlmann T, Wrange O. Inhibition of chromatin assembly in Xenopus oocytes correlates with derepression of the mouse mammary tumor virus promoter. Mol Cell Biol 1991; 11:5259-65.

[46] Chavez S, Beato M. Nucleosome- mediated synergism between transcription factors on the mouse mammary tumor virus promoter. Proc Natl Acad Sci USA 1997; 94:2885-90.

[47] Truss M, Bartsch J, Schelbert A, Hach RJG, Beato M. Hormone induces binding of receptors and transcription factors to a rearranged nucleosome on the MMTV promoter in vivo. EMBO J 1995; 14:1737-51.

[48] Li Q, Wrange O. Translational positioning of a nucleosomal glucocorticoid response element modulates glucocorticoid receptor affinity. Genes and Dev 1993; 7:2471-82.

[49] Li Q, Wrange $\mathrm{O}$. The accessibility of a glucocorticoid response element dependent on its rotational positioning. Mol Cell Biol 1995; 15:4375-84.

[50] Pham TA, McDonnell DP, Tsai M J, O'Malley BW. Modulation of progesterone receptor binding to progesterone response elements by positioned nucleosomes. Biochemistry 1992; 31:1570-8.

[51] Luisi BF, Xu WX, Otwinowski Z, Freedman LP, Yamamoto KR, Sigler P. Crystallographic analysis of the interaction of the glucocorticoid receptor with DNA. Nature 1991; 352:497-502.

[52] Candau R, Chavez S, Beato M. The hormone responsive region of mouse mammary tumor virus positions a nucleosome and precludes access of nuclear factor 1 to the promoter. J Steroid Biochem Mol Biol 1996; 57:19-31. 


\section{Chromatin and transcriptional control}

[53] Blomquist P, Li Q, Wrange O. The affinity of nuclear factor 1 for its DNA site is drastically reduced by nucleosome organization irrespective of its rotational and translational position. $J$ Biol Chem 1996; 271:154-9.

[54] Carr KD, Richard-Foy H. Glucocorticoids locally disrupt an array of positioned nucleosomes on the rat tyrosine aminotransferase promoter in hepatoma cells. Proc Natl Acad Sci USA 1990; 87:9300-4.

[55] Reik A, Schutz G, Stewart AF. Glucocorticoids are required for establishment and maintenance of an alteration in chromatin structure: induction leads to a reversible disruption of nucleosomes over an enhancer. EMBO J 1991; 10:2569-76.

[56] Schmid A, Fascher KD, Horz W. Nucleosome disruption at the PHO5 promoter upon PHO5 induction occurs in the absence of DNA replication. Cell 1992; 71:853-64.

[57] Polach K J, Widom J. A model cooperative binding of eukaryotic regulatory proteins to nucleosomal target sites. J Mol Biol 1996; 258:800-12.

[58] Polach K J, Widom J. Mechanism of protein access to specific DNA sequences in chromatin: a dynamic equilibrium model for gene regulation. J Mol Biol 1995; 254:130-49.

[59] Adams CC, Workman JL. The binding of disparate transcriptional activators to nucleosomal DNA is inherently cooperative. Mol Cell Biol 1995; 15:1405-21.

[60] Kurumizaka H, Wolffe AP. Sin mutations of histone H3: influence on nucleosome core structure and function. Mol Cell Biol 1997; (in press).

[61] Caron F, Thomas JO. Exchange of histone H1 between segments of chromatin. J Mol Biol 1981; 146:513-37.

[62] Louters L, Chalkley R. Exchange of histones H1, H2A and H2B in vivo. Biochemistry 1985; 24:3080-5.

[63] Pennings S, Meersseman G, Bradbury EM. Mobility of positioned nucleosomes on 5S rDNA. J Mol Biol 1991; 220:101-10.

[64] Chipev CC, Wolffe AP. Chromosomal organization of Xenopus laevis oocyte and somatic 5S rRNA genes in vivo. Mol Cell Biol 1992; 12:45-55.

[65] Varga-Weisz PD, Wilm M, Boute E, Dumas K, Mann M, Becker PB. Chromatin remodeling factor CHRAC contains the ATPses ISWI and topoisomerase II. Nature 1997; 388:598-602.

[66] Pazin M J, Kamakaka RT, Kadonaga JT. ATP- dependent nucleosome reconfiguration and transcriptional activation from preassembled chromatin templates. Science 1994; 266:2007-11.

[67] Hayes J J, Clark D J, Wolffe AP. Histone contributions to the structure of DNA in the nucleosome Proc. Natl Acad Sci USA 1991; 88:6829-33.

[68] Yoshinaga SK, Peterson SL, Herskowitz I, Yamamoto KR. Roles of SWI1, SWI2 and SWI3 proteins for transcriptional enhancement by steroid receptors. Science 1992; 258:1598-604.

[69] Muchardt C, Yaniv M. A human homolog of Saccharomyces cerevisiae SNF2/SWI2 and Drosophila brm genes potentiates transcriptional activation by the glucocorticoid receptor. EMBO J 1993; 12:4279-90.

[70] Bresnick EH, Bustin M, Marsaud V, Richard-Foy H, Hager GL. The transcriptionally-active MMTV promoter is depleted of H1. Nucl Acids Res 1992; 20:273-8.

[71] Lee HH, Archer TK. Nucleosome-mediated disruption of transcription factor-chromatin initiation complexes at the mouse mammary tumor virus long terminal repeat in vivo. Mol Cell Biol 1994; 14:32-41.

[72] Bartsch J, Truss M, Bode J, Beato M. Moderate increase in histone acetylation activates the mouse mammary tumor virus promoter and remodels its nucleosome structure. Proc Natl Acad Sci USA 1996; 93:10741-6.

[73] Perlman A J, Stanley F, Samuels HH. Thyroid hormone nuclear receptor: evidence for multimeric organization in chromatin. J Biol Chem 1982; 257:930-8.

[74] Samuels HH, Perlman A J, Raaka BM, Stanley F. Organization of the thyroid hormone receptor in chromatin. Rec Prog Hormone Res 1982; 38:557-99. 


\section{Alan P WOLFFE}

[75] Glass CK, Lipkin SM, Devary OV, Rosenfeld MG. Positive and negative regulation of gene transcription by a retinoic acid- thyroid hormone receptor heterodimer. Cell 1989; 59:697-708.

[76] Leblanc BP, Stunnenberg HG. 9- cis retinoic acid signaling: Changing partners causes some excitement. Genes Dev 1995; 9:1811-6.

[77] Li C, Schwabe JWR, Banayo E, Evans RM. Co- expression of nuclear receptor partners increases their solubility and biological activities. Proc Natl Acad Sci USA 1997; (in press).

[78] Fondell JD, Roy AL, Roeder RG. Unliganded thyroid hormone receptor inhibits formation of a functional preinitiation complex: implications for active repression. Genes and Devel 1993; 7:1400-10.

[79] Baniahmad A, Ha I, Reinberg D, Tsai SY, Tsai M J, O'Malley BW. Interaction of a receptor $\beta$ with transcription factor TFIIB may mediate target gene activation and derepression by thyroid hormone. Proc Natl Acad Sci USA 1993; 90:8832-6.

[80] Le Douarin B, Zechel C, Garnier J-M, Lutz Y, Tora L, Pierrat B, et al. The N-terminal part of TIF1, a putative mediator of the ligand- dependent activation function (AF-2) of nuclear receptors, is fused to B-raf in the oncogenic protein T18. EMBO J 1995; 14:2020-33.

[81] Voegel J J, Heine MJS, Zechel C, Chambon P, Gronemeyer H. A 160 KDa transcription mediator for the ligand-dependent activation function AF- 2 of nuclear receptors. EMBO J 1996; 15:366775.

[82] Kamei Y, Xu L, Heinzel T, Torchia J, Kurokama R, Gloss B, et al. A CBP integrator complex mediates transcriptional activation and AP-1 inhibition by nuclear receptors. Cell 1996; 85:40314.

[83] Fondell JD, Ge H, Roeder RG. Ligand induction of a transcriptionally active thyroid hormone receptor coactivator complex. Proc Natl Acad Sci USA 1996; 93:8329-33.

[84] Horlein A J, Naar AM, Heinzel T, Torchia J, Gloss B, Kurokawa R, et al. Ligand-independent repression by the thyroid hormone receptor mediated by a nuclear receptor co-repressor. Nature 1995; 377:397-404.

[85] Chen JD, Evans RM. A transcriptional co-repressor that interacts with nuclear hormone receptor. Nature 1995; 377:454-7.

[86] Chen JD, Umesono K, Evans RM. SMRT isoforms mediate repression and antirepression of nuclear receptor heterodimers. Proc Natl Acad Sci USA 1996; 93:7567-71.

[87] Chakravarti D, LaMorte V J, Nelson MC, Nakajima T, Juguilon H, Montminy M, Evans, RM. Mediation of nuclear receptor signalling by CBP/p300. Nature 1996; 383:99-103.

[88] Yang X-J, Ogryzko VV, Nishikawa J-I, Howard B, Nakatani, Y. A p300/CBP-associated factor that competes with the adenoviral E1A oncoprotein. Nature 1996; 382:319-24.

[89] Ogryzko VV, Schiltz RL, Russanova V, Howard BH, Nakatani Y. The transcriptional coactivators p300 and CBP are histone acetyltransferases. Cell 1996; 87:953-9.

[90] Onate SA, Tsai SY, Tsai M- J, O'Malley BW. Sequence and characterization of a coactivator for the steroid hormone receptor superfamily. Science 1995; 270:1354-7.

[91] Alland L, Muhle R, Hou Jr H, Potes J, Chin L, Schreiber-Agus N, et al. Role of NCoR and histone deacetylase in Sin3- mediated transcriptional and oncogenic repression. Nature 1997; 387:49-55.

[92] Heinzel T, Laviusky RM, Mullen TM, Soderstrom M, Laherty CD, Torchia JT, et al. N- CoR, mSIN3, and histone deacetylase in a complex required for repression by nuclear receptors and Mad. Nature 1997; 387:43-8.

[93] Nagy L, Kao HY, Chakravarti D, Lin R J, Hassig CA, Ayer DE, et al. Nuclear receptor repression mediated by a complex containing SMRT, mSin3A, and histone deacetylase. Cell 1997; 89:37380.

[94] Hebbes TR, Thorne AW, Crane-Robinson C. A direct link between core histone acetylation and transcriptionally active chromatin. EMBO J 1988; 7:1395-402.

[95] Hebbes TR, Thorne AW, Clayton AL, Crane- Robinson C. Histone acetylation and globin gene switching. Nucl Acids Res 1992; 20:1017-22. 


\section{Chromatin and transcriptional control}

[96] Hebbes TR, Clayton AL, Thorne AW, Crane-Robinson C. Core histone hyperacetylation comaps with generalized DNase I sensitivity in the chicken $\beta$ - globin chromosomal domain. EMBO J 1994; 13:1823-30.

[97] Tazi J, Bird A. Alternative chromatin structure at CpG islands. Cell 1990; 60:902-20.

[98] Lee DY, Hayes J J, Pruss D, Wolffe AP. A positive role for histone acetylation in transcription factor binding to nucleosomal DNA. Cell 1993; 72:73-84.

[99] Ura K, Kurumizaka H, Dimitrov S, Almouzni G, Wolffe AP. Histone acetylation: influence on transcription by RNA polymerase, nucleosome mobility and positioning, and linker histone dependent transcriptional repression. EMBO J 1997; 16:2096-2107.

[100] Brownell JE, Zhou J, Ranalli T, Kobayashi R, Edmondson DG, Roth SY, Allis CD. Tetrahymena histone acetyltransferase A: a homolog to yeast Gcn5p linking histone acetylation to gene activation. Cell 1996; 84:843-51.

[101] Janknecht R, Hunter T. A growing coactivator network. Nature 1996; 383:22-3.

[102] Taunton J, Hassig CA, Schreiber SL. A mammalian histone deacetylase related to a yeast transcriptional regulator Rpd3. Science 1996; 272:408-11.

[103] Yang WM, Inouye C, Zeng Y, Bearss D, Soto E. Transcriptional repression by YY1 is mediated by interaction with a mammalian homolog of the yeast global regulator RPD3. Proc Natl Acad Sci USA 1996; 93:12845-50.

[104] Shi Y, Seto E, Chang L-S, Shenk T. Transcriptional repression by YY1, a human GL1- Kruppelrelated protein, and relief of repression by adenovirus E1A protein. Cell 1991; 67:377-88.

[105] Shrivastava A, Calame K. An analysis of genes regulated by the multi-functional transcriptional regulatory Yin yang 1 . Nucl Acids Res 1994; 22:5151-5.

[106] Ayer DE, Lawrence QA, Eisenman RN. Mad- Max transcriptional repression is mediated by termary complex formation with mammalian homologs of yeast repressor Sin3. Cell 1995; 80:76776.

[107] Laherty CD, Yang WM, Sun JM, Davie JR, Seto E, et al. Histone deacetylase associated with the mSin3 corepressor mediate Mad transcriptional repression. Cell 1997; 89:349-56.

[108] Yoshida M, Kijima M, Akita M, Beppu T. Potent and specific inhibition of mammalian histone deacetylase both in vivo and in vitro by Trichostatin A J. Biol Chem 1990; 265:17174-9.

[109] Wong J, Shi Y-B, Wolffe AP. A role for nucleosome assembly in both silencing and activation of the Xenopus TR $\beta$ A gene by the thyroid hormone receptor. Genes Dev 1995; 9:2696-711.

[110] Wong J, Shi Y-B, Wolffe AP. Determinants of chromatin disruption and transcriptional regulation instigated by the thyroid hormone receptor: hormone regulated chromatin disruption, is not sufficient for transcriptional activation. EMBO J 1997; 16:3158-71.

Received 27-10-1997. Accepted 4-11-1997. 